\title{
Un enfoque filosófico de la vejez y algunas consideraciones bioéticas
}

\author{
A philosophical approach to old age and some bioethical \\ considerations
}

\author{
Lourdes Velázquez
}

\author{
Centro Interdisciplinario de Bioética, Universidad Panamericana, Ciudad de México, México \\ Recibido el 16 de enero de 2020; aceptado el 17 de febrero de 2020 \\ Disponible en Internet el 22 de abril de 2020
}

\section{Resumen}

La prolongación de la vida humana es uno de los rasgos característicos de nuestra era tecnológica y determina un escenario para cuestionamientos filosóficos, algunos de los cuales empiezan a encontrar respuestas en el campo de la Bioética. Uno de los más importantes es la denominada institucionalización en los cuidados prolongados o confinamiento, y se plantea entonces si se trata de una opción bioéticamente correcta. Una apreciación positiva de la vejez es parte de las raíces mismas de la cultura occidental; sin embargo, en las sociedades tecnológicamente avanzadas comienza a cargarse con matices negativos: lejos de otorgar un valor positivo a la vejez, se ha dado forma a un nuevo mito antiético: el del hombre «joven», «productivo». La sociedad suele privar a los ancianos de casi todas las obligaciones, relegándoles cuando su trabajo ya no es deseado El enfoque bioético ante el paciente geriátrico privilegia el respeto que se le debe como ser humano por encima de la lástima, la compasión o la caridad. Algo parecido puede decirse de la hospitalización, que ha demostrado tener efectos negativos sobre la capacidad funcional de los ancianos. En particular esa práctica aumenta uno de los factores más negativos en la vida del anciano, es decir, la soledad. Para combatir dicha soledad existen algunas posibilidades, la primera de las cuales es la de poner a la persona anciana en contacto con

Correo electrónico: 1velazquezg@up.edu.mx 
otras personas. Además, hay nuevos instrumentos para combatir la soledad del adulto mayor: la risoterapia y la robótica social. De estos damos una breve descripción, discutiendo también las condiciones bioéticamente relevantes de su adopción.

(C) 2020 Centros Culturales de México, A.C. Publicado por Masson Doyma México S.A. Todos los derechos reservados.

Palabras clave: Envejecimiento; Filosofía de la viejez; Soledad del anciano; Risoterapia; Robótica social

Abstract: The prolongation of human life is one of the characteristic features of our technological era and establishes a scenario for philosophical questions, some of which begin to find answers in the field of Bioethics. One of the most important is the so-called institutionalisation in prolonged care, or confinement, and the question is whether this is a bioethically correct option. A positive assessment of old age is part of the very roots of Western culture, however in technologically advanced societies it begins to be accompanied by negative nuances. Far from giving a positive value to old age, a new unethical myth has surfaced: that of the «young», «productive» man. Society often deprives the elderly of almost all obligations, relegating them when their work is no longer desired. The bioethical approach to the geriatric patient grants the respect owed to them as human beings instead of pity, compassion or charity. Something similar can be said of hospitalisation, which has been shown to have negative effects on the functional capacity of the elderly. In particular, this practice increases one of the most negative factors in the life of the elderly, loneliness. To combat this loneliness there are some possibilities, the first of which is to put the elderly person in contact with other people. In addition, there are new tools for combatting the loneliness of the elderly: laughter therapy and social robotics. A brief description of these is presented, as a well a discussion on their adoption.

(C) 2020 Centros Culturales de México, A.C. Published by Masson Doyma México S.A. All rights reserved.

Keywords: Aging; Philosophy of old age; Loneliness of the elderly person; Laughter therapy; Social robotics

\section{Introducción}

El crecimiento, el desarrollo, la maduración y el envejecimiento son fases en la continuidad de la vida de la célula, los tejidos, los órganos y el organismo entero. El envejecimiento tiene un origen multifactorial, es una característica intrínseca fundamental de todo ser vivo y se caracteriza principalmente por la pérdida gradual de la eficiencia operacional, la vitalidad y la resistencia al estrés.

La prolongación de la vida humana y el aumento en la probabilidad de alcanzar una edad avanzada han resultado rasgos característicos de nuestra era tecnológica; 
con ellos se ha propiciado, además del cambio en la pirámide demográfica en muchas poblaciones, con acumulación creciente de individuos de edad avanzada, una distinta visión de las etapas finales de la existencia humana y un aumento en el número de enfermos crónicos.

Todos estos hechos ayudan a conformar un escenario para cuestionamientos filosóficos nunca antes enfrentados por la humanidad, algunos de los cuales empiezan a encontrar respuestas en el campo de la Bioética.

Y es que la necesidad de atención a los ancianos es verdaderamente inmensa, ya que el envejecimiento demográfico, como lo hemos mencionado, ha puesto en evidencia que no existen suficientes recursos materiales, ni tampoco humanos, que ayuden a resolver satisfactoriamente este fenómeno que se ha convertido en problema.

En la actualidad la población de viejos está predominando en los países desarrollados, por lo cual la estructura social de atención a sus necesidades básicas se ha ido incrementando y esta situación ha provocado una verdadera transformación en todo el mundo, lo que incluye cambios en el orden político, el económico, las políticas de salud, la demanda de pensiones, etc., problema que también está sucediendo en los llamados países en desarrollo, como México.

La Organización de las Naciones Unidas definió en 1980 que los 60 años de edad y más marcan el inicio de la denominada etapa de la vejez. En base a ello, hay en el mundo más de 416 millones de ancianos. Para el año 2030 los mayores de 65 años serán el 21,1\% de los habitantes del planeta. De este total de ancianos, el $72 \%$ vivirán en los países en desarrollo, y se espera que la esperanza de vida al nacimiento en Latinoamérica se incremente de los 64,1 que se tuvieron durante 1985 a los 71,8 para el año 2030 (García, 2004:28-35).

Las cifras anteriores permiten apreciar que la situación demográfica está cambiando de manera importante, por lo que, además de los problemas que enfrentan los países en desarrollo, comparten también con los países avanzados el reto del envejecimiento de su población.

De aquí se puede inferir que la problemática de las decisiones sobre este grupo de personas se ha vuelto y se pondrá más complicada, por lo que las soluciones rápidas a la población sin meditar sobre los beneficios a las personas serán motivos de graves injusticias y errores que provocarán, a su vez, costos muy altos, en especial sobre las vidas humanas. 
Uno de los elementos que merece una especial atención es la denominada institucionalización en los cuidados prolongados o confinamiento, y se plantea entonces si se trata de una opción bioéticamente correcta, que no se aleje del bien integral del anciano. La edad cronológica no debería ser un parámetro que determine su bien integral como persona, sobre todo si, como hemos señalado, la edad limitante para considerar a alguien viejo son los 65 años de edad; este es un límite del todo arbitrario, ya que no se toman en cuenta los parámetros biológicos para su definición cronológica, sino más bien se refiere a un contexto socioeconómico para sustentarlo con un fundamento utilitario y pragmático en el cual el deseo de bienestar individual y social se construye mediante el cálculo del costo/beneficio, lo cual no me parece resulte lo más adecuado. En otras palabras, para considerar un bien integral en un sujeto anciano hay que ir más allá de la concepción cronológica y biológica del individuo como persona humana.

\section{Significado del término vejez}

Hacer un resumen de los matices semánticos que el término «vejez» ha adquirido en el desarrollo de nuestra cultura es sin duda alguna una labor compleja que implica un estudio mucho más profundo que el que ofreceremos a continuación; sin embargo, podemos darnos una idea recorriendo los pasos que han marcado - y marcan en cada cultura - nuestra postura en relación con el tiempo «individual», por así decirlo, o con el tiempo de la edad de la vida. Tal camino no puede, por supuesto, dejar de lado la consideración de la percepción universal del Tiempo, ya que es precisamente sobre la base de este, y en relación con él y su posición en el ámbito social, que el hombre se concibe a sí mismo como un «convertirse en» y no solo como un «estar en» (autorrepresentación espacial).

En efecto, el hombre no se reconoce en el plano temporal como la expresión de una evolución «histórica» que implicaría un desarrollarse (concepto vinculado a una conciencia diferente del ser en el tiempo). Más bien se concibe como un caminante según el ritmo de la vida, por lo que su paso desde la infancia hasta la vejez es ciertamente una progresión inevitable, pero principalmente «natural», desde el florecimiento hasta el deterioro y la muerte.

Por consiguiente, percibe la existencia como «ser en el presente» y no como «ser en el futuro»; por lo tanto, acepta las transformaciones biológicas y las registra - sin connotarlas- con valores morales o funciones sociales. 
El término vejez proviene del latín; su origen etimológico se encuentra en el vocablo vetus, que se traduce como «viejo», por lo que la vejez sería la cualidad de viejo, alguien de edad avanzada o algo antiguo y que no es nuevo. $Y$ hace referencia a la senectud o edad senil (Pérez Porto y Merino, 2010:1).

\section{Modelos sociales y culturales}

Para el ser humano, la vejez es el período de la decadencia: se ve a sí mismo apagado, encogido, tartamudo, enfermo, vulnerable a resfriados, a tos y a miles de cambios en su estado de ánimo, siendo estas manifestaciones signos evidentes de la proximidad a la muerte. En épocas pasadas incluso hubo quienes afirmaron que el envejecimiento era una enfermedad en sí misma. Es decir, reconocían ya lo que hoy llamamos síndromes geriátricos y la condición de fragilidad del anciano (Rodríguez García y Lazcano Botello, 2004). Al mismo tiempo, no siente el peso de la carga moral (como si fuera el testigo de las virtudes de antaño o, al revés, de la máscara tragicómica de los vicios humanos); tampoco se siente marginado o anulado. Viviendo el tiempo en un sentido cualitativo («tiempo bueno o malo»), se siente la vejez como una pérdida de fuerzas vitales, pero no como un proceso de pérdida alienante de las funciones sociales. La serenidad con la que enfrenta la decadencia es el espejo de una sociedad con ritmos lentos pero fluidos, una sociedad que vive en simbiosis con la naturaleza externa y que da sentido a la existencia individual reconociéndola como parte integral de la repetición cíclica de las estaciones (Minois, 1988:37).

Una apreciación positiva de la vejez es parte de las raíces mismas de la cultura occidental, como da fe una de las obras más famosas de la literatura latina: el $D e$ senectute («Sobre la viejez»), escrito por Marco Tulio Cicerón en el siglo primero antes de Cristo (Cicerón, 2001) En esta obra, Cicerón imagina un diálogo entre el célebre Catón el Viejo (conocido también como «el Censor»), llegado a los ochenta y cuatro años, y dos jóvenes patricios romanos: Escipión, hijo de Pablo Emilio, y su amigo Lelio. El diálogo es introducido por las palabras de Escipión, que expresan el asombro suyo y de Lelio frente a la serenidad con la cual Catón está viviendo su vejez y la intensa actividad desplegada por el octogenario. Por lo tanto, Catón empieza una tranquila argumentación y examina las más comunes críticas que se le hacen a la viejez y da sus razones para no renegar de la misma y aceptarla como una etapa más de la vida, mediante ejemplos sacados de la historia griega y romana. Los cargos analizados son: la debilidad y la decadencia física, la disminución de las capacidades intelectuales, la imposibilidad de gozar de los placeres de los sentidos, la rareza del carácter y la avaricia. En el desarrollo del diálogo se discuten en 
profundidad filosófica grandes temas como el de la muerte y de la posible inmortalidad después de la muerte, cuales se podían enfocar antes del surgimiento de la perspectiva judeocristiana.

Muy diferente a la relación que el hombre establece con la idea de la vejez, cuando con el cambio de los modelos sociales y culturales abandona la idea del «tiempo natural» y comienza a considerar la temporalidad como el flujo cuantitativo y, por lo tanto, medible o fragmentado en segmentos-unidad de valor constante. Abandonadas las formas de la mentalidad de la sociedad agraria tradicional y con el surgimiento de la civilización urbana, la noción del tiempo se transforma: del tiempo cíclico, en el que las diferencias entre el pasado, presente y futuro eran extremadamente sutiles y por lo tanto relativas, se pasa al tiempo lineal, concebido como una recta que toca el presente y va del pasado al futuro. Obviamente la transición entre estas dos formas de conceptualización del tiempo surgió gradualmente, sin un desprendimiento neto, ya que en una cultura siempre coexisten diferentes ritmos sociales correspondientes a la conciencia de los grupos individuales, más allá del modelo temporal dominante impuesto por la clase que tiene el control de la sociedad misma. Sin embargo, «lo que queremos destacar en este contexto es cómo en el mundo occidental dicha transformación del concepto del tiempo se lee bajo el esquema cada vez más presente de la consciencia de la brevedad (y no fugacidad o caducidad) de la vida. Esto se manifestó a raíz de la caída de la cultura feudal, donde el tiempo se percibió como algo capitalizable que hay que evaluar, medir, guardar y explotar» (Pinna y Pott, 2011:28).

\section{La longevidad como valor positivo}

Es en este contexto que el concepto de vejez comienza progresivamente a cargarse con matices negativos. En contraste con la imagen del anciano patriarca, poseedor de la tradición y de la sabiduría, comienza por primera vez a manifestarse, pasando por un proceso de vinculación entre edad y actividad, la figura del viejo sedentario dedicado al estudio y a la reflexión, de lo que emerge la idea del anciano improductivo que se convierte en una carga para una sociedad dominada por las leyes del capitalismo. Si en los tiempos modernos la «tercera edad» todavía se concebía como una etapa con oportunidades de trabajo y desempeño que otorgaban valores educativos y de conservación, en la sociedad contemporánea parece haber prevalecido, más allá de los matices lingüísticos hipócritas y con falsos principios en sus declaraciones, el proceso de negativización del concepto de la vejez. De hecho, hoy podemos decir que somos testigos de una paradoja aún más trágica. 
La longevidad, lejos de otorgar un valor positivo a la vejez, ha dado forma a un nuevo mito antiético: el del hombre «joven», «productivo», encargado de la tarea de vivificar y capitalizar una sociedad emergente hoy en día fosilizada. Un mito detrás del cual no es difícil ver la incapacidad de vivir una relación natural y serena con la evolución biológica. Es decir, la fobia a la decadencia del cuerpo, el miedo a la muerte, aislar de la sociedad a la persona destinándola a consumirse en la soledad «no escandalosa» de los hospitales y asilos.

De estos horrores el anciano es tanto víctima como actor: una víctima, porque él es testigo del proceso natural de decadencia y, por lo tanto, un elemento para marginar, para eliminar; actor, porque está obligado a usar la máscara de «bien conservado» (una especie de momificación macabra en la vida) hasta que la muerte no lo libere. No solo eso, pertenece a una cultura que se basa en la filosofía del «tiempo es dinero», donde lo social ha sido absorbido por la «comunidad global» y donde los medios de comunicación se presentan como los únicos portadores de valores y «recuerdos», lo que no solo lo expropia del papel natural de la memoria histórica de una sociedad, sino que lo aprisiona en una jaula temporal cuyo límite ad quem es el paso a la edad de la jubilación, es decir, el momento en el que el hombre alcanza la fase improductiva de su vida.

Una vez fuera del mundo del trabajo, este hombre no tiene muchas alternativas: o «rejuvenece» al convertirse en un sustituto de padres ausentes y patéticamente trata de no dejarse consumir por los signos del envejecimiento, o se somete a una soledad que lo lanza hacia la senescencia, volviéndose más degradante cuanto más los otros se percatan de su inminente retroceso a la infancia. Una vez más, es evidente como del concepto de vejez se mide el grado de vida efectiva de una sociedad. Máxima expresión del tiempo.

«En cuanto a la categoría del tiempo y la finitud, la insistencia de Séneca es vivir bien cada día y no proyectar hacia el futuro nuestras expectativas. El vivir bien no es, entonces, ni acumular honores ni riquezas que, además de ser inestables, alteran el ánimo. La vejez no tiene como meta acumular bienes para disfrutarlos cuando ya pasó la mayor parte de la existencia ante nosotros. Así, en la perspectiva de Séneca, el vivir bien no se equipara a la noción de calidad de vida que se maneja contemporáneamente. Vivir bien es asumir la racionalidad del mundo decretado por Dios y aprender a sobrellevar las carencias o abundancias, manteniendo el ánimo en tranquilidad» (Gómez Álvarez, 2019: 29). 


\section{La esperanza de vida activa}

El tema del envejecimiento pocas veces se comenta y es de poco interés en nuestra sociedad «orientada hacia la juventud». Actualmente se han alcanzado grandes avances en salud, gracias a los progresos de la tecnología y de la medicina, y la esperanza de vida se ha incrementado notablemente.

Pero si bien la esperanza de vida se ha elevado, esto no ha ocurrido de forma paralela con la «esperanza de vida activa», definiendo esta como la parte de los últimos años de la vida de la persona que puede vivir sin depender de terceras personas. Gran parte de las incapacidades que se observan en los ancianos dependen de enfermedades inducidas por el estilo de vida, y varios autores piensan que elevar la esperanza de vida quizá no sea la meta principal de investigación del anciano, sino más bien evitar o retrasar la incapacidad que surge como resultado del estilo de vida previo. Muchos ancianos llegan así a la tercera edad como discapacitados (León Correa, 2009:39).

Si se entiende por «discapacidad» la disminución temporal o permanente de la capacidad funcional, biológica, psicológica o social del individuo para desempeñar sus actividades cotidianas en forma útil e independiente, el anciano no es, por el solo hecho de haber alcanzado una edad avanzada, un discapacitado. En este sentido conviene distinguir el proceso eugérico del proceso patogérico, de modo que solo este último representa la verdadera discapacidad.

Lo que sí constituye un hecho incontrovertible es que la probabilidad de enfermar - y por tanto perder la capacidad funcional para desarrollar las actividades cotidianas de manera independiente y útil— se incrementa con la edad y que el número de discapacitados es mayor entre los ancianos que entre otros grupos de edad. En un estricto sentido, podemos decir que el abordaje bioético de la discapacidad del anciano corresponde con el de la discapacidad del enfermo.

\section{La discapacidad de origen social}

La discapacidad de los individuos de edad avanzada frecuentemente es más de origen social que biológico o psicológico. Los ancianos que logran evitar o superar limitaciones físicas y mentales tienen que enfrentar no solo la soledad y el aislamiento, sino en ocasiones hasta el maltrato y el confinamiento. «En un estudio realizado en 
personas de más de 90 años, cuya salud era razonable y que podrían atender con relativa independencia a sus actividades diarias, solo un poco más de la mitad de ellos apreciaban su longevidad. Uno de cada seis deseaba la muerte. Su existencia perdió valor o sentido al morir su cónyuge o sus hijos, carecían de interés y relaciones sociales y tenían pocos motivos para seguir viviendo» (Loya García, 1996:111).

La sociedad suele privar a los ancianos de casi todas las obligaciones, relegándoles cuando su trabajo ya no es deseado, ni se les pide consejo, ni se aprecia su aportación y experiencia. Los enfoques con los que la sociedad ha enfrentado sus relaciones con los ancianos han sido irrespetuosos y han propiciado la discapacidad. Los objetivos de favorecer la independencia, mejorar la autoestima y estimular la función no se han visto favorecidos con los abordajes basados en el paternalismo. Existen ancianos que solicitan trabajo aunque no sea renumerado y hasta pagando ellos porque se les emplee.

El enfoque bioético ante el paciente geriátrico privilegia el respeto que se le debe como ser humano por encima de la lástima, la compasión o la caridad. La integración social es una aspiración a la que no han podido responder la mayoría de las sociedades occidentales de hoy en día y que solo han recurrido a soluciones artificiosas. La sociedad, pues, no solo ha sido incapaz de resolver el problema del anciano discapacitado, sino que frecuentemente es el origen mismo de la discapacidad.

Hay muchos indicios de que varios de los cambios físicos de los ancianos que se tienden a atribuir al proceso de envejecimiento se deben, en realidad, a la acumulación de años de desuso, lo que ubica aún a la discapacidad física en un contexto social.

\section{La hospitalización}

Algo parecido puede decirse de la hospitalización que ha demostrado tener efectos negativos sobre la capacidad funcional de los ancianos ya que al término de la hospitalización el anciano suele estar más discapacitado que al inicio, aun cuando se haya logrado una mejoría en la enfermedad física. Esto coloca «entre las responsabilidades éticas del personal de salud, no solo evitar la hospitalización de los ancianos siempre que sea posible, sino también evitar el deterioro funcional que la hospitalización con lleva y, en su caso, indicar las medidas de rehabilitación del anciano tampoco es una idea fácilmente aceptada cuando se parte del punto de vista utilitario» (Loya García, 1996:112). 


\section{Enfoque utilitario}

En las sociedades occidentales contemporáneas orientadas a la producción de bienes y servicios se da una tendencia a establecer categorías de los seres humanos en función de su capacidad de contribuir a esta producción. En esta óptica, el enfermo se convierte no solo en un parásito sino en un verdadero estorbo, pues no solo deja de contribuir a la producción sino que puede hasta limitar la capacidad productiva de quienes lo rodean. Gran parte de las acciones en servicios de salud no cuentan con la orientación altruista que se pensaría deberían tener, sino que se orientan a preservar o a establecer lo antes posible la planta productiva de la sociedad. Incluso el término «discapacidad», aunque tiene una connotación más amplia, suele relacionarse con la imposibilidad de realizar un trabajo. Cuando el paciente es joven este efecto es más claro, porque también lo es que tiene un porcentaje mucho más alto de reincorporarse a la producción, sobre todo si tiene un padecimiento curable y si se le suministran oportunamente las medidas terapéuticas adecuada. En cambio, el anciano enfermo, en el mejor de los casos, contará con una probabilidad muy restringida de reincorporarse al proceso productivo, en función de su incapacidad orgánica para superar la enfermedad y de su limitada esperanza de vida (Lifshitz Guinzberg, 1994:309-312).

Resulta del todo obvio que las ideas expuestas no suelen confesarse abiertamente, pues, al considerar a los seres humanos como recursos del proceso productivo, contrastan con los enfoques humanistas que las sociedades modernas ponderan aunque no siempre practican, y desafortunadamente estas ideas influyen de manera definitiva en la atención que se proporciona hoy en día a los ancianos enfermos. El paciente geriátrico no solo ha dejado de ser útil temporalmente en razón de su enfermedad, sino que tiene relativamente pocas probabilidades de ser y de sentirse útil, se le suele enfocar no solo en función de su valor relativo y, en este sentido, tiene una clara desventaja en relación con los enfermos más jóvenes. No suele observarse, por ejemplo, la misma actitud ante el paciente con cáncer en fase terminal cuando se atiende a un joven que a un anciano en las mismas circunstancias, y la actuación del personal en este último caso se guía más bien por sentimientos de compasión o de lástima.

«Por lo que es importante vivir bien, no importando la duración en el tiempo. Tener la muerte enfrente no es propio de la vejez, sino de toda la vida. La vida se vive día a día, y en eso la vejez no es diferente de la juventud» (Gómez Álvarez, 2019:9). 


\section{La soledad en el adulto mayor}

Es muy conocido y no necesita de documentación el hecho de que la soledad es una amenaza muy fuerte para la salud de las personas ancianas, sea desde el punto de vista psicológico, sea desde el punto de vista de la salud física misma, ya que la soledad puede fácilmente inducir a un estado de depresión. Y la depresión, además de ser una grave patología psicológica, puede llevar a condiciones muy severas y hasta inducir en el adulto mayor el deseo de la muerte y de esta manera abrir el camino hacia la eutanasia Para combatir dicha soledad existen algunas posibilidades, la primera de las cuales y que resulta la más evidente es la de poner a la persona anciana en contacto con otras personas. Que sean mayores o que sean más jóvenes no importa, lo importante es estar en grupo, lo cual no siempre es fácil (Rodríguez Martín, 2009).

Y un primer punto que deseaba analizar en esta estancia de investigación era precisamente el de ver cómo se pueda disfrutar la posibilidad, cuando exista, de hacer convivir a la persona mayor en un grupo, como de hecho sucede ya en el CESA (Centro Diurno para ancianos Alberto Sordi), un lugar donde en primera persona pude constatar que el anciano es valorado y recibe ayuda para seguir creciendo como persona. Y en sus instalaciones, conjuntamente a la atención médico sanitaria, se lleva a cabo también una labor de carácter social que fomenta la convivencia a través de una compañía de teatro, un taller de alta costura, clases de yoga, canto, musicoterapia, excursiones culturales, paseos por la naturaleza, vacaciones de verano y muchas cosas más que favorecen el trabajo en equipo.

Sin embargo, el puro y simple hecho físico del estar en un grupo de por sí no brinda ninguna ventaja si no se añade alguna razón para estar en el grupo, y la razón espontánea que más o menos todos hemos experimentado alguna vez en nuestra vida es la del hecho de encontrarse para conversar y sobre todo para reírnos juntos.

\section{Nuevos instrumentos para combatir la soledad del adulto mayor}

\section{Risoterapia}

Mientras que la mayoría de adultos mayores se hallan rodeados de compañeros afines, extrañan el contacto familiar. Las terapias de risa tienen el poder de llegar más allá de la curación del cuerpo. La risa hace que las relaciones con la gente se vuelvan más fuertes, lo que disipa el sentimiento de soledad. Las personas mayores 
pueden disfrutar mucho de sesiones diarias de risoterapia, ya que genera un sentido de pertenencia, cercanía y fraternidad, mejora la movilidad y la comunicación personal y trae armonía a las relaciones interpersonales

Además de ser un remedio para la enfermedad, la risa produce pensamientos positivos, relaja, reduce las tensiones: ayuda a salir de la depresión emocional y brinda una perspectiva positiva de las cosas Posiblemente todos nos acordamos de alguna jornada aburrida porque el clima era malo y estábamos juntos intentando pasar el tiempo contando chistes, y el hecho de contar chistes nos hacía pasar varias horas sin darnos cuenta del hecho de que estábamos en una jornada aburrida; aumentaba el buen humor porque cada uno escuchaba el chiste del otro y se reía, y hasta los más tímidos se arriesgaban a contar algún chiste.

Esta experiencia muy normal nos ayuda a entender la importancia de una verdadera terapia que ha venido desarrollándose en los últimos años, que es la terapia de la risa, llamada también risoterapia, la cual ha sido experimentada con mucho éxito, sobre todo en el caso de niños. Sabemos que cuando al niño enfermo, por ejemplo, se le rodea de una atmósfera de risa se puede obtener una reducción muy fuerte de los síntomas subjetivos y hasta del dolor. Los beneficios de la risoterapia en el caso de los niños nos hacen plantear una pregunta bastante lógica: ¿por qué no intentar aplicar la risoterapia también en los adultos mayores? No esperamos conseguir todos los resultados que podemos alcanzar en el caso de los niños, pero podrán ser igualmente muy positivos y en particular ayudar a luchar contra la soledad, aparte de las ventajas de tipo físico, en particular fisiológicas, que de por sí la risa produce en el organismo humano (Velázquez, 2015:138).

Sin embargo, sabemos que muchas personas ancianas no viven en comunidades como el CESA en las que sea posible formar grupos de ancianos que se junten para reírse y someterse a un tratamiento mediante la risa. De hecho, muchísimos ancianos viven solos en su propia casa, y los jóvenes del día de hoy a menudo no quieren conversar con gente de su familia. Entonces para estos ancianos el problema de la soledad es particularmente duro y no todos pueden permitirse aliviar esa soledad gracias a la compañía de otra persona que no sea de la familia, sino que sea un cuidador o una cuidadora. En estos casos, la dificultad de la soledad se manifiesta bajo otros aspectos que no pueden ser aliviados mediante la risoterapia, ya que se trata de exigencias más concretas y materiales que podríamos calificar como necesidad de ser atendidos y cuidados. 


\section{Robótica social}

Desde otro punto de vista, la ayuda nos puede venir de la robótica social, uno de los aspectos que más tiempo lleva intentando destacar dentro del campo de ese tipo de máquinas. Su función es clara: crear aparatos o dispositivos al alcance de las personas para mejorar su vida, es decir, su salud física y mental.

Todos sabemos que en los últimos años se han construido muchos robots para uso médico, en la rehabilitación y hasta para hacer operaciones quirúrgicas muy delicadas y complicadas. Sin embargo, hay también otros robots que han sido desarrollados más recientemente con fines funcionales y que presentan características muy semejantes a las operaciones que puede realizar una persona humana (humanoides) hacia un paciente anciano, no solamente en el sentido de que si se trata de un paciente enfermo puede ayudarle en algunas terapias, sino también en el caso de un paciente en buena salud física que se encuentre solo y pueda recibir ayuda en su actividades o simplemente contar con una forma de compañía, como es el caso de robots que pueden plantearle ciertas preguntas, saludarle, preguntarle cómo se encuentra y hacer muchísimas otras cosas. En este caso lo llamamos paciente porque la soledad de por sí es ya un inicio de patología del paciente hacia el alivio de no sentirse solo, y la presencia del robot le da confort y ayuda a combatir la sensación de soledad. De hecho, sabemos que para muchos ancianos una ayuda de cierta importancia es tener un televisor continuamente encendido para escuchar y para ver. Tener a su lado un robot que pueda darles la sensación de una compañía es sin duda hasta más importante.

Todas las ventajas mencionadas arriba no pueden ocultar algunos problemas éticos relacionados con el uso de estos robots. Un robot normalmente tiene un precio elevado y esto plantea un problema de justicia social, ya que solo una minoría de personas ricas podría comprarlo y tenerlo a su servicio, mientras la gran mayoría de la población anciana seguiría en la soledad. Por consiguiente, no sería esta la solución correcta del problema de la soledad de los ancianos. A esta objeción se contesta subrayando que el costo de los robots ha disminuido rápidamente en los últimos años y seguirá bajando con el aumento de su producción, y que además podría ser compensado por el hecho de ahorrar en los gastos que de todas maneras implica el servicio de un cuidador humano. Esto se ve claramente en países como Japón, que se encuentra a la vanguardia en cuanto a la creación y utilización de este tipo de robots y que en ello han encontrado una solución al problema de la escasez de personas 
que puedan ser cuidadores y al mismo tiempo evitan la utilización de mano de obra extranjera que los japoneses no admiten fácilmente en su país (Beliz, 2018:2).

El segundo aspecto ético depende del hecho de que un robot al final de cuentas es una máquina, y entonces este uso del robot aumenta un hecho que ya es típico de la medicina actual, es decir, que el enfermo se encuentra con máquinas y máquinas y máquinas, y esto disminuye el contacto con los demás seres humanos, lo que es malo desde muchos puntos de vista. Se trata de un problema real, y algunos autores ya han subrayado la necesidad de complementar el uso de los robots con particulares cuidados a que esto no se convierta en una forma de deshumanización, en el sentido de que el adulto mayor no identifique poco a poco su entorno normal con un entorno de máquinas.

\section{Conclusiones}

La proximidad de la muerte impone su modulación a las consideraciones éticas que rodean la atención de los ancianos. Se ha llegado incluso a decir que «un enfermo joven puede morir, pero un enfermo viejo tiene que morir». Las difíciles decisiones ante el paciente terminal que caracterizan a nuestra época adquieren la matriz que impone la relativamente corta expectativa de vida aun si el padecimiento terminal se resolviera, y la consideración de que está ocurriendo el evento precisamente al término de una vida y no como un hecho accidental. Aunque no es fácil sustraerse a estas consideraciones: al implicar el enfoque bioético que jerarquiza la autonomía, el paciente anciano resulta equiparable con el paciente de otras edades.

El anciano resulta un discapacitado en la medida en que las enfermedades que caracterizan a esta etapa de la vida generen disfunción. La responsabilidad social no es solo de proporcionar la atención médica que el anciano requiere, sino tomar en cuenta la necesidad de propiciar su independencia, su incorporación a la vida productiva y el respeto que merece como ser humano y persona.

Hay que reconocer que el valor relativo de un individuo es solo un artificio social, puesto que varía según diferentes sociedades; son muy conocidos los ejemplos de grupos sociales que conceden una preponderancia a sus ancianos, de tal manera que las jerarquías se invierten. En un enfoque bioético se considera a los seres humanos en razón de su valor intrínseco, del respeto que se les debe como personas y al margen de su valor relativo. 
El anciano puede redimirse solo en un contexto que no le haga temer el futuro, que en sus años productivos sepa alternar sabiamente el tiempo biológico, que siempre encuentre un momento para detenerse, escuchar y volver a la infancia.

Debemos construir una cultura a favor de un envejecimiento saludable. Es el tiempo de las culturas nómadas que tal vez deberíamos redescubrir: culturas en las que al anciano se le confiaba la tarea de narrar y reconstruir, entre ellos, los mitos, los acontecimientos épicos, los recuerdos individuales. Pero sobre todo culturas en las que el niño y el viejo se encuentran y construyen ese lenguaje universal de memoria y arte que da vida a los sueños, temores y esperanzas de la humanidad.

\section{Fondos}

Ministerio de la ciencia y tecnología de España. Proyecto Internacional de investigación MINECO/FEDER FFI2015-64529-P.

\section{Conflicto de intereses}

Los autores declaran no tener ningún conflicto de intereses

\section{Agradecimientos}

A raíz de una estancia de investigación en la Universidad Campus Biomédico de Roma como profesora visitante tuve la oportunidad de estar en el CESA (Centro para la salud del anciano de la fundación Alberto Sordi), gracias al cual fue posible parte de la elaboración de este trabajo, ya que uno de los objetivos principales consistía en la propuesta de instrumentos que puedan ayudar a combatir la soledad en la vejez. Va mi agradecimiento de manera particular al Prof. Vittoradolfo Tambone y a la Dra. Maddalena Pennacchini, directora del Centro por el apoyo y facilidades brindadas, sin los cuales difícilmente hubiera sido posible realizar el presente trabajo.

\section{Bibliografía}

Beliz, Gustavo (2018). Colaboremos para enfrentar los riesgos éticos de la inteligencia artificial. Disponible en: https://blogs.iadb.org/conocimiento-abierto/es/riesgos-eticos-de-la-inteligencia-artificial/.

Cicerón Marco Tulio. (2001). De senectute. Madrid: Triacastela.

García, J. (2004). Perfil epidemiológico del adulto mayor en México. In Antología para la unidad temática de salud en el anciano. México, Departamento de Salud Pública: Facultad de Medicina, UNAM. 
Gómez Álvarez, J. E. (2019). Reflexiones bioéticas acerca de la ancianidad desde el estoicismo de Séneca. Revista de Medicina y Ética, 31(1), 13-29. http://dx.doi.org/10.36105/mye.2020v31n1.01

León Correa, F. J. (2009). Bioética razonada y razonable. Santiago de Chile: Fundación Interamericana Ciencia y Vida.

Lifshitz Guinzberg, A. (1994). Bioética ante el paciente geriátrico discapacitado. Revista de Medicina IMSS, 32, 309-312.

Loya García, B. E. (1996). Bioética en el paciente anciano. In Bioética: temas selectos. México: Cuéllar.

Minois, G. (1988). Storia della vecchiaia - Dall'Antichitá al Rinascimento. Italia: Laterza.

Pérez Porto, Julián, y María Merino. Definición de vejez. 2010 [actualizado 2013]; [consultado 6 Feb 2018]. Disponible en: https://definicion.de/vejez/.

Pinna, G. y Pott, H.-G. (2011). Senilitá. Immagini della vecchiaia nella cultura occidentale. Italia: Edizioni dell'Orso.

Rodríguez García, R. M. C. y Lazcano Botello, G. A. (2004). Práctica de la Geriatría. México: McGraw-Hill. Rodríguez Martín, M. (2009). La soledad en el anciano. Gerokomos., 20(4), 192-199 [consultado 11 Feb 2020]. Disponible en: http://scielo.isciii.es/scielo.php?script=sci_arttext\&pid=S1134-928X2009000400003.

Velázquez, L. (2015). El poder terapéutico de la risa. Bioethics Update, 1(2), 130-142. 